Received: 3 November 2017

Accepted: 14 February 2018 Published: 26 February 2018
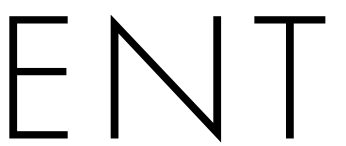
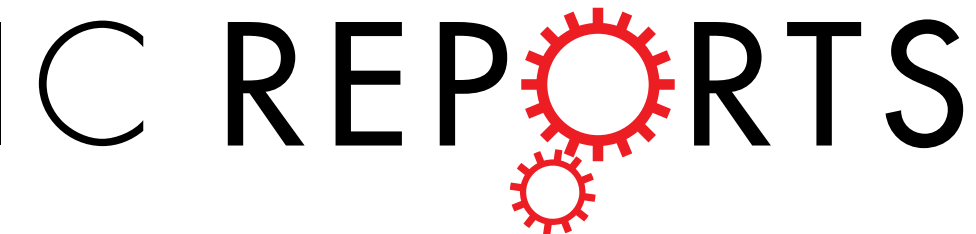

RETRACTED ARTICLE

\title{
OPEN Antibiotic growth promoters
} virginiamycin and bacitracin methylene disalicylate alt r the chicken intestinal metabu bome

\author{
Ujvala Deepthi Gadde ${ }^{1}$, Sungtaek Oh $\mathbb{D}^{1}{ }^{1}$, Hyun S. Lillehoj ${ }^{1}$ \& L 'P. Lillehoj ${ }^{2}$
}

Although dietary antibiotic growth promoters have lo. hee ...ead to increase growth performance in commercial food animal production, the biochemical a ils associated with these effects remain poorly defined. A metabolomics approach was us to chara erize and identify the biochemical compounds present in the intestine of broiler chic it.. I a standard, unsupplemented diet or a diet supplemented with the antibiotic growth promote ", virginiamycin or bacitracin methylene disalicylate. Compared with unsupplemented contrals the level. of 218 biochemicals were altered (156 increased, 62 decreased) in chickens given the rginı cin-supplemented diet, while 119 were altered (96 increased, 23 decreased) with the $b_{a}$ acin- spplemented diet. When compared between antibioticsupplemented groups, 79 cher ıcals wt Vtered (43 increased, 36 decreased) in virginiamycin- vs. bacitracin-supplemented ch en The ct.anges in the levels of intestinal biochemicals provided a distinctive biochemical s; vat unid e to each antibiotic-supplemented group. These biochemical signatures were chara rized by -reases in the levels of metabolites of amino acids (e.g. 5-hydroxylysine, 2-- mir tipate, 5-hydroxyindoleaceate, 7-hydroxyindole sulfate), fatty acids (e.g. oleate/vaccenat - , ticosape. énoate, 16-hydroxypalmitate, stearate), nucleosides (e.g. inosine, $\mathbf{N}^{6}$ methyladeno ne), and vitamins (e.g. nicotinamide). These results provide the framework for future studies to ide ify natu al chemical compounds to improve poultry growth performance without the use of in-feed a. : ics.

average commercial broiler consumes $3.2 \mathrm{~kg}$ of feed over 35 days to achieve $1.8 \mathrm{~kg}$ of body weight, compared wit more nan $20 \mathrm{~kg}$ of feed over 112 days to attain the same weight in the $1920{ }^{\prime} \mathrm{s}^{1}$. This improvement in poultry own.. erformance has been achieved, in large part, through advances in animal genetics, health, and nutrition, 1. ding the use of in-feed antibiotic growth promoters such as virginiamycin and bacitracin methylene disalicyla.e. Dietary antibiotics have been used in the food animal industry for more than 60 years, not only to control infectious diseases, but also to increase feed efficiency and improve growth performance ${ }^{2,3}$. In chickens, subtherapeutic, in-feed antibiotics can increase body weight gain up to $8 \%$ and decrease the feed conversion ratio (feed intake/body weight gain) up to $5 \%$, both compared with an antibiotic-free diet ${ }^{4}$. However, use of antibiotic growth promoters in food animal production has led to the development of antibiotic resistance among the commensal gut microflora, thus increasing the zoonotic risk such as potential to be transferred to humans ${ }^{5-8}$.

The mechanisms through which dietary antibiotics exert their growth promoting effects remain to be established. Antibiotics were originally thought to improve animal growth through reductions in the number and diversity of the normal bacterial flora present in the gut, which in turn, increased the bioavailability of nutrients available to the host and/or reduced the production of microbial metabolites deleterious to animal growth ${ }^{9-13}$. Alternatively, antibiotics were suggested to improve growth performance through an anti-inflammatory effect directed toward the intestinal epithelium ${ }^{14}$. With the advent of novel molecular biology and bioinformatics techniques, it is now clear that changes in the host intestinal inflammatory response ${ }^{15-18}$, as well as the structure and

${ }^{1}$ Animal Bioscience and Biotechnology Laboratory, Beltsville Agricultural Research Center, Agricultural Research Service, United States Department of Agriculture, Beltsville, MD, 20705, USA. ${ }^{2}$ Department of Pediatrics, University of Maryland School of Medicine, Baltimore, MD, 21201, USA. Ujvala Deepthi Gaddea and Sungtaek Oh contributed equally to this work. Correspondence and requests for materials should be addressed to H.S.L. (email: Hyun. Lillehoj@ars.usda.gov) 


\begin{tabular}{|c|c|c|c|c|}
\hline & & \multicolumn{2}{|c|}{ Predicted Group } & \multirow[b]{2}{*}{ Class Error } \\
\hline & & Virginiamycin & Control & \\
\hline \multirow{2}{*}{ Actual Group } & Virginiamycin & 6 & 1 & 0.143 \\
\hline & Control & 1 & 6 & 0.143 \\
\hline \multicolumn{5}{|c|}{ Predictive Accuracy $=85.7 \%$} \\
\hline & & \multicolumn{2}{|c|}{ Predicted Group } & \multirow{2}{*}{ Class Error } \\
\hline & & Bacitracin & Control & \\
\hline \multirow{2}{*}{ Actual Group } & Bacitracin & 6 & 1 & 0.143 \\
\hline & Control & 2 & 5 & 0.143 \\
\hline \multicolumn{5}{|c|}{ Predictive Accuracy $=78.5 \%$} \\
\hline & & \multicolumn{2}{|c|}{ Predicted Group } & \multirow{2}{*}{ Class Error } \\
\hline & & Virginiamycin & Bacitracin & \\
\hline \multirow{2}{*}{ Actual Group } & Virginiamycin & 5 & 2 & 0.285 \\
\hline & Bacitracin & 3 & 4 & 0.427 \\
\hline \multicolumn{5}{|c|}{ Predictive Accuracy $=65.0 \%$} \\
\hline & & \multicolumn{2}{|c|}{ Predicted Group } & \\
\hline & & Control & Vir + BMD & \\
\hline \multirow{2}{*}{ Actual Group } & Control & 5 & 2 & \\
\hline & Vir + BMD & 2 & 12 & 0.143 \\
\hline \multicolumn{4}{|c|}{ Predictive Accuracy $=81.0 \%$} & \\
\hline
\end{tabular}

Table 1. Random Forest Analysis of the 30 most significant'y alt ed biochemicals distinguishing between the virginiamycin $v s$. control, bacitracin $v s$. control, and vir. iar analysis of 7 independent samples.

diversity of the gut microbial community ${ }^{19-28}$, occur wi en antibiotics are introduced into animal diets. Based on these studies, dietary antibiotic suppleme no wy pothesized to promote an optimal and balanced microbiota with reduced capacity to evoke a inflan atory response and increased efficiency of energy harvest from nutrients ${ }^{29,30}$.

In a mouse model of antibiot $c$ growth on otion, administration of dietary antibiotics altered the composition and metabolic capabilit of $\mathrm{t}$ e gut $\mathrm{m}$, crobiota by selecting for bacterial species capable of metabolizing complex carbohydrates to hor ain fa cy acids, thus extracting a higher proportion of available calories for energy expenditure ${ }^{31}$. Sv' sequently ${ }^{1}$ et al..$^{32}$ reported that exposure of mice to antibiotics early in life induced long-term metabolic $\epsilon$ fec $y$ accele ating the development of a normal, age-related microbiota. However, definitive linkage of particular gu acterial populations to intestinal metabolic changes remains to be established ${ }^{33}$. The current stu $y$ was underta, en to characterize the combined host- and microbiome-derived metabolic alterations in the cl cken gut following dietary antibiotic supplementation to identify potential chemical metabolites that might be $\mathrm{u} d$ in liev of dietary antibiotics to improve poultry growth performance.

\section{Result}

Effect of dy $y$ antibiotics on broiler growth performance. Dietary supplementation with $20 \mathrm{~g} / \mathrm{ton}$

e broa d spectrum antibiotic, virginiamycin, increased chicken body weight gain by $10.1 \%$ between days 0 and 21 age co npared with chickens fed an unsupplemented diet $(\mathrm{p}<0.05)$. Similarly, chickens fed a diet containing of the narrow spectrum antibiotic, bacitracin methylene disalicylate, had $7.9 \%$ greater body weight gain vared with birds given an unsupplemented diet $(\mathrm{p}<0.05)$.

Effect of dietary antibiotics on intestinal global metabolite levels. A total of 706 biochemicals were identified in the intestinal contents of chickens fed an unsupplemented, control diet, or a diet supplemented with virginiamycin or bacitracin methylene disalicylate. In the virginiamycin $v s$. control groups, the levels of 156 chemicals were increased and 62 were decreased; in the bacitracin $v s$. control groups, 96 chemicals were increased and 23 were decreased; in the bacitracin vs. virginiamycin groups, 43 chemicals were increased and 36 were decreased; and in the control $v s$. both antibiotics groups, 132 chemicals were increased and 46 were decreased.

Metabolite signatures and biochemical importance analyses. Table 1 lists the Random Forest Analysis (RFA) data for metabolite signatures and biochemical importance of the 30 most statistically significantly altered metabolites for distinguishing the virginiamycin $v s$. control, bacitracin $v s$. control, and virginiamycin $v s$. bacitracin groups. RFA of the virginiamycin $v s$. control groups gave a predictive accuracy of $85.7 \%$, while that of bacitracin $v$ s. control groups was $78.5 \%$. Among 7 samples tested from each dietary group, 6 samples from both the virginiamycin and bacitracin groups were predicted to belong to their respective group, while the remaining sample was predicted to belong to the control group. Of 7 control group samples, one was predicted to belong to the virginiamycin group and two were predicted to belong to the bacitracin group. By contrast, RFA of the virginiamycin $v$ s. bacitracin groups gave a predictive accuracy of $65.0 \%$, suggesting that when compared with each other, dietary supplementation with either antibiotic produced a less characteristic biochemical signature compared with the antibiotic vs. control comparisons. Among the biochemicals classified as the most biochemically important for distinguishing between the 3 dietary groups, metabolites of amino acids (33.0\%), fatty acids 
A

B
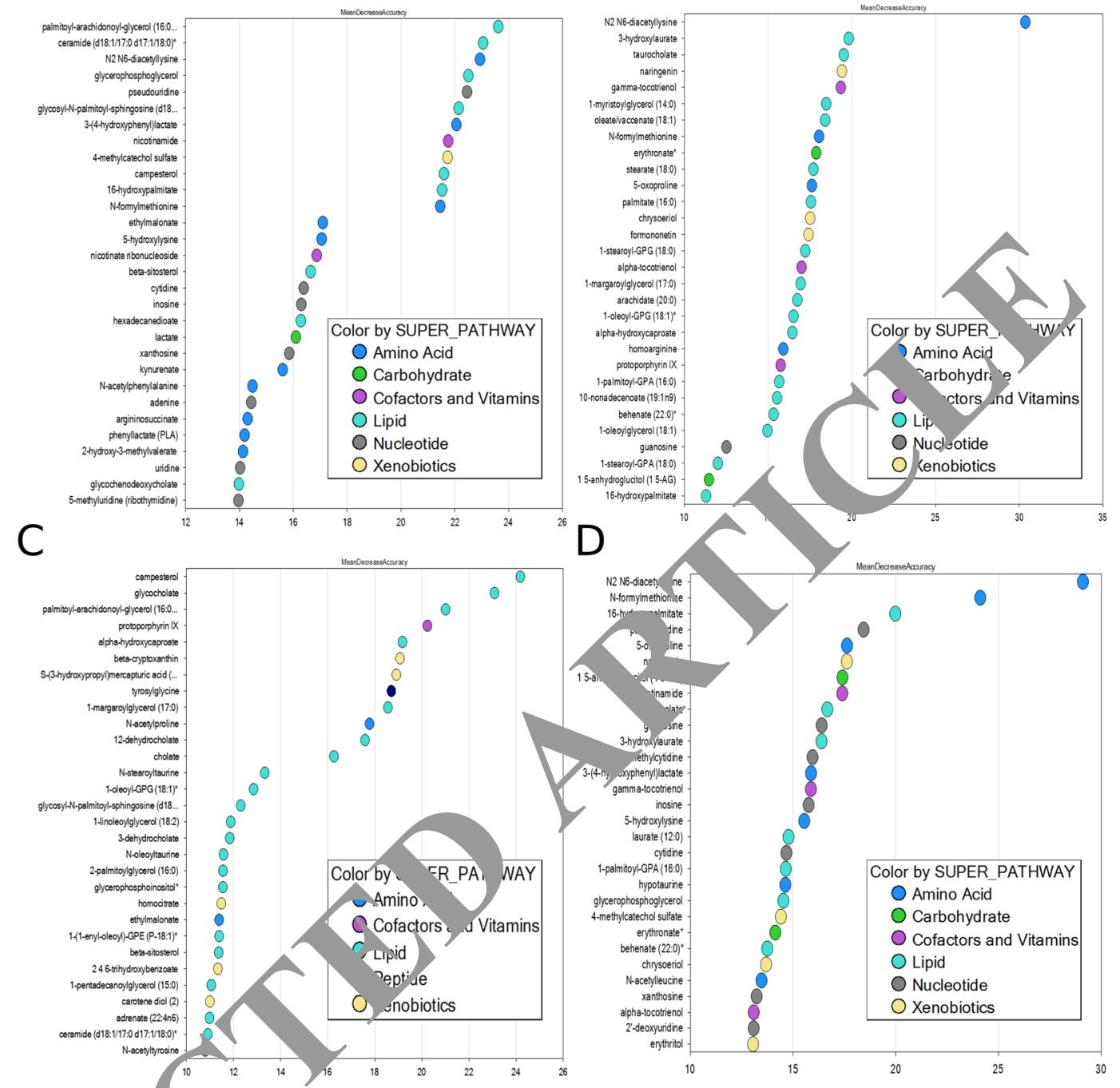

Figure 1. Top biocherhicals whose levels were increased in the virginiamycin $v s$. control (A), bacitracin methylene disalı. s. control (B), virginiamycin $v s$. bacitracin methylene disalicylate (C) and control vs. both iotics dietary groups (D). Biochemicals are listed from bottom to top in increasing order of importance on tributing to the biochemical signatures separating the antibiotic-supplemented groups from insup lemented controls (A-D) or separating the virginiamycin group from the bacitracin group (C), and are lotted $\Lambda$ color-coded symbols according to chemical classification.

(30.0\%), and nucleosides $(23.3 \%)$ accounted for the majority of biochemicals in the virginiamycin $v s$. control groups (Fig. 1A), whereas lipids accounted for $56.7 \%$ and $66.7 \%$ of the biochemicals in the bacitracin $v s$. control (Fig. 1B), virginiamycin vs. bacitracin (Fig. 1C) groups and control vs. both antibiotics (Fig. 1D) respectively.

Specific alterations in amino acid, fatty acids, nucleoside, and nicotinamide metabolites following dietary antibiotic supplementation. Among the amino acids most highly elevated in the virginiamycin $v s$. control and bacitracin $v$ s. control groups were metabolites of lysine and tryptophan. Specifically, levels of the lysine metabolites $\mathrm{N}^{6}$-formyllyisne, 5-hydroxylysine, and 2-aminoadipate were increased 1.25-, 3.07-, and 2.35-fold in the intestinal contents of chicken fed the virginiamycin-supplemented diet compared with unsupplemented controls, while these same biochemicals were increased 1.28-, 2.60-, and 2.70-fold in bacitracin-treated chickens compared with controls. The tryptophan-associated metabolites kynurenine and 5 -hydroxyindoleacetate were increased 1.73- and 1.65-fold in the virginiamycin $v s$. control groups, and 3.02- and 3.22-fold in the bacitracin vs. control groups (Fig. 2A). By contrast, indolelactate levels in virginiamycin- and bacitracin-supplemented chickens were reduced to $18.0 \%$ and $42.0 \%$ of the levels in unsupplemented controls. The levels of other tryptophan metabolites, such as kynurenate (3.00-fold increase), xanthurenate (2.43-fold increase), and 7-hydroxyindole sulfate (4.80-fold increase), were augmented in the virginiamycin $v s$. control groups, but unchanged in the bacitracin $v s$. control groups. 

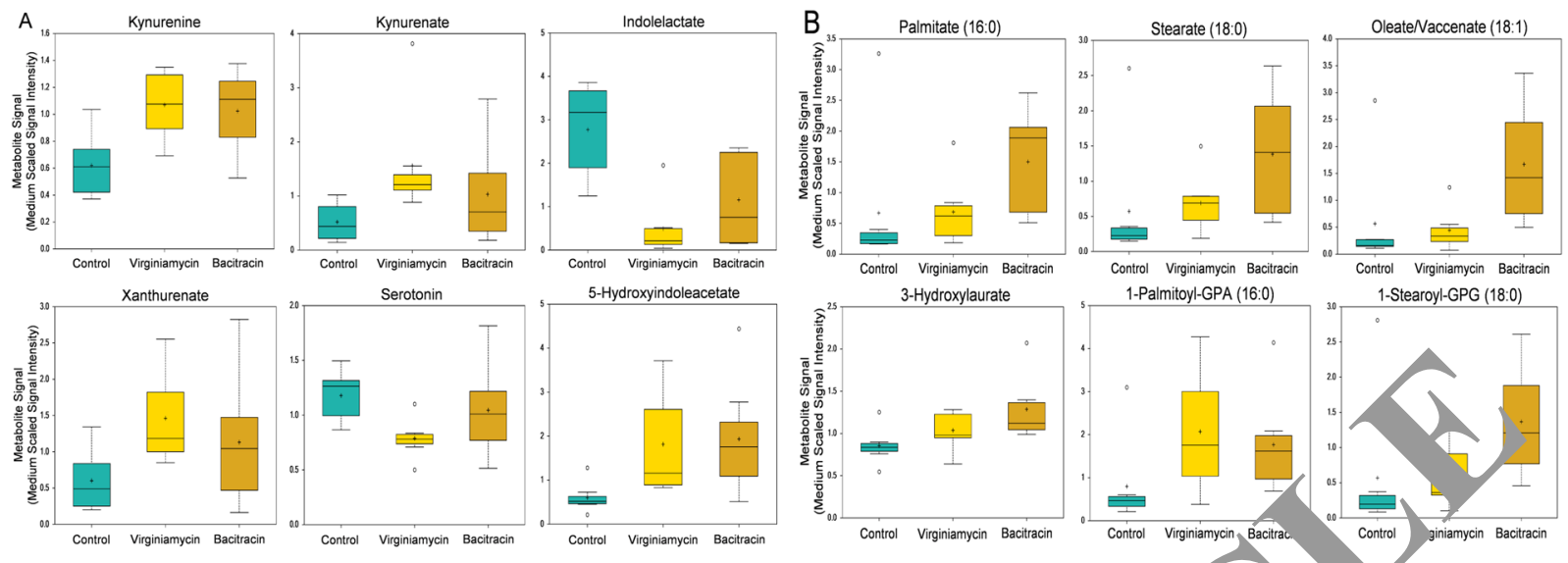

1-Stearoyl-GPG (18:0)
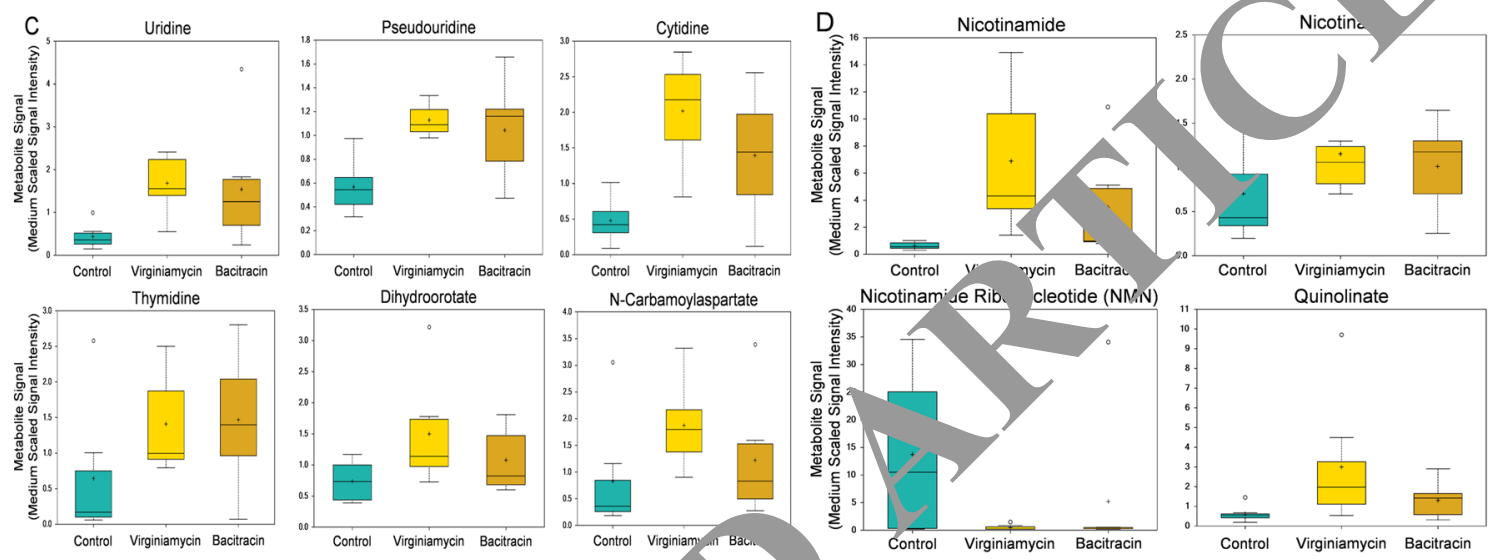

Figure 2. Box-and-whisker plot of the le of netabolites of (A) tryptophan, (B) fatty acids, (C) nucleotides, and (D) nicotinamide in the int stine of ch ns fed an unsupplemented, control diet (green), or a diet supplemented with virginiamy (s llow' or bacitracin methylene disalicylate (brown). The box represents the interquartile range (IQR) Aerinea the $25^{\text {th }}$ and $75^{\text {th }}$ percentiles. The horizontal line represents the medium value. The cross represe, the mean alue. The upper whisker represents Q3 + $(1.5 \times \mathrm{IQR})$, while the lower whisker represents $\mathrm{Q},-(1<\mathrm{IQR})$. Circles represent outliers.

Fatty acids a their metabolites also contributed to the biochemical signatures separating chickens given the anti tic-suppremented diets, particularly the bacitracin-supplemented group, from unsupplemented controls (Fig "ny long chain saturated and polyunsaturated fatty acids, as well as several lysophospholipids, wore incresed in the bacitracin vs. control groups. Most notable in this comparison were oleate/vaccinate (18:1) (2. -fold ncrease), eicosapentaenoate (2.55-fold increase), 16-hydroxypalmitate and stearate (both 2.42-fold in , arachidate (2.39-fold increase), 10-nonadecenoate (2.30-fold increase), palmitate (2.24-fold increase), 3-hydroxylaurate (1.51-fold increase).

lochemicals associated with purine and pyrimidine metabolism that were increased in the virginiamycinor bacitracin-supplemented diets $v s$. unsupplemented controls included inosine (16.7- and 9.23-fold increases, respectively), N-methyl adenosine (14.6-, 11.4-fold increases), 5-methyl uridine (8.04-, 5.29-fold increases), xanthosine (8.18-, 5.73-fold increases), cytidine (4.22-, 2.91-fold increases), uridine (3.86-, 3.53-fold increases), and pseudouridine (1.99-, 1.84-fold increases) (Fig. 2C). Other nucleoside metabolites were increased only in the virginiamycin $v s$. control comparison, including 5,6-dihydrothymine (2.27-fold increase), N-carbamoylaspartate (2.26-fold increase), and dihydroorate (2.03-fold increase). The levels of nicotinamide were increased in the virginiamycin $v s$. control (10.8-fold increase) and bacitracin $v s$. control (5.45-fold increase) groups, whereas its metabolites quinolinate (6.06-fold increase) and nicotinate (1.62-fold increase) were elevated only in virginiamycin vs. control groups (Fig. 2D). Nicotinamide ribonucleotide (NMN) levels in both virginiamycin- and bacitracin-supplemented chickens were reduced to levels $<10 \%$ of the unsupplemented controls.

\section{Discussion}

Virginiamycin and bacitracin methylene disalicylate are common growth enhancers used in the poultry industry. Virginiamycin is a streptogramin antibiotic produced by Streptomyces virginiae as a mixture of two macrocyclic lactone peptolides, virginiamycin $\mathrm{M}$ and virginiamycin $\mathrm{S}$, both of which bind to the bacterial $50 \mathrm{~S}$ ribosomal subunit to synergistically inhibit protein synthesis ${ }^{4}$. Virginiamycin $\mathrm{M}$ is a polyunsaturated cyclic peptolide while virginiamycin $S$ is a cyclic hexadepsipeptide ${ }^{34}$. Dietary supplementation of chickens with virginiamycin decreased intestinal colonization by Clostridium perfringens ${ }^{35}$, and decreased the severity and mortality due to necrotic enteritis caused by C. perfringens ${ }^{36}$, both compared with unsupplemented controls. Bacitracin is a mixture of 
more than 10 related cyclic peptides produced by Bacillus subtilis and B. licheniformis that disrupt bacterial cell wall synthesis by inhibiting dephosphorylation of lipid pyrophosphate ${ }^{4}$. Dietary supplementation of chickens with bacitracin reduced gut colonization by C. perfringens and Enterococcus faecalis ${ }^{19,37}$, but increased the number of Salmonella enterica, compared with unsupplemented controls ${ }^{38}$. Compared with chickens fed an unsupplemented diet, intestinal microbiome analyses of chickens fed virginiamycin- and/or bacitracin-supplemented diets have generally revealed a decreased in microbial diversity, with an increase in Enterococcus and Lactobacillus spp., although a decreased frequency of $L$. salivarius has been noted ${ }^{19,20,24,25,27,28}$. Other investigators have reported an altered bacterial composition, but no change in gut microbiome richness or diversity, associated with virginiamycin- or bacitracin-supplemented diets, compared with antibiotic-free diets ${ }^{22,39}$.

The levels of amino acid metabolites, particularly those of lysine and tryptophan, were substantially altered by dietary supplementation with virginiamycin or bacitracin methylene disalicylate. Tryptoph? 11 is metabolized by two major pathways, either through kynurenine leading to niacin and associated cof? rrs, including nicotinamide adenine dinucleotide (NAD), or through a series of indole-related compounds le $\mathrm{g}$ to rotonin and melatonin. Dietary supplementation with either virginiamycin or bacitracin methylene. 1 cylate increased the levels of kynurenine, as well as its metabolites, kynurenate and quinolj, in the chicken gut. Kynurenine and kynurenate play important roles in the regulation of inflammation 'nd adap immune response, as well as multiple neurological pathways ${ }^{40,41}$. Increased activity of the ky urenine $\mathrm{pa}_{a}$ ay is internally consistent with decreased levels of indolelactate and serotonin following antibic c supplementation. Serotonin (5-hydroxytryptamine) receptors are found throughout the intestinal ileum and ociated smooth muscle ${ }^{42}$. In the small intestine, serotonin enhances the rate at which intestinal content ove a on the digestive system. Increased body weight gain in antibiotic-supplemented diets might be clated, art, through decreased serotonin levels leading to increased residence time and absorption of intes: 1 nutrien. Consistent with the increased levels of quinolinate following antibiotic supplementation, nicotin imic etabolism and the NAD biosynthetic pathway were also shown to be increased in chickens given th virginian in-or bacitracin-containing diets. Interestingly, $\mathrm{NMN}$, which is produced from nicotinamide, vas c creased in virginiamycin-supplemented birds, with a trend for decreased levels following bacitracin supp suggesting that nicotinamide might be shuttled to nicotinate biosynthesis. Indeed, animals in the vir increased nicotinate levels compared with unsupplei nted cont. is.

One of the most striking features of the current aa. in the increase in levels of many long chain fatty acids, particularly polyunsaturated fatty acids (PUFAs), in the intestine of bacitracin-supplemented, but not virginiamycin-supplemented, chickens. PUFAs are not commonly found in bacteria, and while chickens can synthesize PUFAs from dietary linolenate $n \mathrm{nd}$. ate, much of the PUFA content in chicken tissues is thought to originate from ingested sources ${ }^{43}$. Inch deve of PUFAs in the ileum of bacitracin-supplemented birds, therefore, might be the result of decreac $d$ int al a ssorption. PUFAs are important as substrates for inflammatory and anti-inflammatory fatty aci ${ }^{1}$, such as $t_{1}$ prostaglandins, leukotrienes, and thromboxanes ${ }^{44}$. Omega- 3 fatty acids with a $\mathrm{C}=\mathrm{C}$ double bona ${ }^{t} \mathrm{~b}$. thir ${ }^{\prime}$ carbon atom from the end of the carbon chain, such as eicosapentaenoate, are thought to hav more a inf ammatory properties, while omega- 6 fatty acids, such as arachidonate, contribute to inflamm. reactiol. Increased levels of eicosapentaenoate following antibiotic supplementation in the current tudy ds support to the non-antibiotic, anti-inflammatory theory of antibiotic growth promotion $^{14}$. Thr se growth-r ed metabolites are shown in Kyoto encyclopedia of genes and genomes pathway (KEGG) and $\mathrm{h}$ man metabolome database (HMDB), further studies are required to summarizing important/ abundant met olites pa hway in chickens. In summary, this study compared the metabolome profiles of the intestinal conte of $\mathrm{cr}$ ickens fed an unsupplemented diet with animals given a diet containing the antibiotic growth moters virginiamycin or bacitracin methylene disalicylate. The results demonstrated that antibiotic supplem ntw ad profound effects on the levels of a wide variety of chemical metabolites, particularly amino a-ids, fatt acids, nucleosides, and nicotinamide-related compounds. Further, these altered metabolite levels provic $d$ a bio bemical signature unique to each antibiotic supplementation group when compared with unsupple$\mathrm{m}$ ontrols. Future investigations of the chemical compounds identified in this study might provide new roaches to enhance food animal growth without the use of antibiotics.

\section{Methods}

Animals and ethics statement. Forty-five-day-old commercial broiler chickens (Ross/Ross, Longenecker's Hatchery, Elizabethtown, PA) were housed in electrically-heated battery starter cages (Petersime, Gettysburg, $\mathrm{OH})$. Chickens were raised in starter cages until 14 days of age and transferred to finisher cages where they were kept until the end of the experimental period. Feed and water were provided ad libitum. Animal husbandry followed guidelines for the care and use of animals in agricultural research ${ }^{45}$. All experimental protocols were approved by the Small Animal Care Committee of the Beltsville Agricultural Research Center.

Experimental diets and intestinal metabolomics analysis. Chickens ( $n=15$ /group) were fed from hatch with a corn- and soybean meal-based unsupplemented, basal diet (control) formulated to meet or exceed the National Research Council's nutrient requirements for broiler chickens ${ }^{46}$, or the basal diet supplemented with $20 \mathrm{~g} /$ ton (22 ppm) virginiamycin (Phibro Animal Health, Teaneck, NJ) or 50 g/ton (55 ppm) bacitracin methylene disalicylate (Zoetis, Durham, NC) (Table 2). Body weights and feed conversion ratios were measured daily until day 21 . At 3 weeks of age, 7 chickens/group were euthanized by cervical dislocation and the intestinal ileum harvested. Ileal content was collected by gently fingers-stripping the ileal segment. Intestine contents were collected aseptically, immediately placed on dry ice, and stored at $-80^{\circ} \mathrm{C}$. Global metabolomic profiling of the intestinal contents was performed by mass spectrometry (MS) (Metabolon, Durham, NC) as described ${ }^{47-49}$. Raw data was extracted and processed using the DiscoveryHD $4^{\mathrm{TM}}$ global metabolomics platform. Compounds were identified by comparison to library entries of purified standards or recurrent unknown entities based on retention index, 


\begin{tabular}{|l|c|}
\hline Ingredient & $\%$ \\
\hline Corn & 55.78 \\
\hline Soybean meal & 37.03 \\
\hline Soybean oil & 2.97 \\
\hline Dicalcium phosphate & 1.80 \\
\hline Calcium carbonate & 1.51 \\
\hline Salt & 0.38 \\
\hline Poultry Vitamin Mix ${ }^{\mathrm{a}}$ & 0.22 \\
\hline Poultry Mineral Mix ${ }^{\mathrm{b}}$ & 0.15 \\
\hline DL-Methionine & 0.10 \\
\hline Choline-chloride, $60 \%$ & 0.06 \\
\hline Total & 100 \\
\hline Calculated values (dry matter basis) & \\
\hline Crude protein & 24.00 \\
\hline Calcium & 1.20 \\
\hline Available Phosphorus & 0.51 \\
\hline Lysine & 1.40 \\
\hline Methionine & 0.49 \\
\hline Cysteine + Methionine & 0.80 \\
\hline True metabolizable energy (TMEn), kcal/kg & \\
\hline
\end{tabular}

Table 2. Diet composition. ${ }^{a}$ Vitamin mixture provided the follo hg nutrients per kg of diet: vitamin A, 2,000 $\mathrm{IU}$; vitamin D3, $22 \mathrm{IU}$; vitamin E, $16 \mathrm{mg}$; vitamin $\mathrm{K}, 0.1 \mathrm{mg}$ tap $3.4 \mathrm{mg}$; vitamin $\mathrm{B} 2,1.8 \mathrm{mg}$; vitamin B6, $6.4 \mathrm{mg}$; vitamin B12, $0.013 \mathrm{mg}$; biotin, $0.17 \mathrm{mg}$; pantothen. cid, $8.7 \mathrm{mg}$; folic acid, $0.8 \mathrm{mg}$; niacin, $23.8 \mathrm{mg}$. ${ }^{\mathrm{b}}$ Mineral mixture provided the following nutrients $\mathrm{p}-\mathrm{kg}$ of diet. ¿, $0.4 \mathrm{mg} ; \mathrm{Zn}, 0.22 \mathrm{mg} ; \mathrm{Mn}, 0.18 \mathrm{mg}$; Co, $0.0013 \mathrm{mg} ; \mathrm{Cu}, 0.021 \mathrm{mg}$.

accurate mass match to the library $\pm 1 \quad \mathrm{pm}$, a $\mathrm{MS} / \mathrm{MS}$ forward and reverse scores between experimental data and authentic standards. MS/MS srores ba d on comparison of the ions present in the experimental spectrum to the ions present in the li'orary spec

Statistical analysis. A t $\mathrm{v}$. ailed student's t-test was used to compare body weight gains and feed conversion ratios of $c^{1}$ ickens to $1 \mathrm{e}$ unsupplemented and virginiamycin- and bacitracin methylene disalicylate-supplem nte iets. ANOVA was used to identify the biochemicals whose levels were significantly altered among the three die groups (virginiamycin $v s$. control, bacitracin methylene disalicylate $v s$. control, virginiamycin $y$. bacitracin $m$,thylene disalicylate) following median scaling, log transformation, and imputation of missing alues, if any, with the minimum value observed for each compound. Standard statistical analyses of log-transfor d data were performed using Array Studio software (OmicSoft, Cary, NC). For analyses that were nct standar. . ray Studio, the programs R (R Foundation for Statistical Computing, Vienna, Austria) or JMP (SA_itute, Cary, NC) were used. Changes in biochemical levels with $\mathrm{p} \leq 0.05$ were considered statistically sign fic ant. An estimate of the false discovery rate (FDR) was obtained by calculating the q-value to account he false positives that normally occur in metabolomics-based studies. Random Forest Analysis (RFA) was pes armed oy computing the Mean Decrease Accuracy (MDA) as a measure of biochemical importance to a tssmu.tion.

\section{Re,erences}

1. Diarra, M. S. \& Malouin, F. Antibiotics in Canadian poultry productions and anticipated alternatives. Front. Microbiol. 5 (2014).

2. Dahiya, J. P., Wilkie, D. C., Van Kessel, A. G. \& Drew, M. D. Potential strategies for controlling necrotic enteritis in broiler chickens in post-antibiotic era. Anim. Feed Sci. Technol. 129, 60-88 (2006).

3. Castanon, J. I. R. History of the use of antibiotic as growth promoters in European poultry feeds. Poult. Sci. 86, 2466-2471 (2007).

4. Butaye, P., Devriese, L. A. \& Haesebrouck, F. Antimicrobial growth promoters used in animal feed: effects of less well known antibiotics on gram-positive bacteria. Clin. Microbiol. Rev. 16, 175-88 (2003).

5. Aarestrup, F. M. Occurrence, selection and spread of resistance to antimicrobial agents used for growth promotion for food animals in Denmark. APMIS, Acta Pathol. Microbiol. Immunol. Scand. 108, 1-48 (2000).

6. van den Bogaard, A. E. \& Stobberingh, E. E. Epidemiology of resistance to antibiotics: links between animals and humans. Int. J. Antimicrob. Agents 14, 327-335 (2000).

7. Phillips, I. et al. Does the use of antibiotics in food animals pose a risk to human health? A critical review of published data. J. Antimicrob. Chemother. 53, 28-52 (2004).

8. Lekshmi, M., Ammini, P., Kumar, S. \& Varela, M. F. The food production environment and the development of antimicrobial resistance in human pathogens of animal origin. Microorganisms 5, 11 (2017).

9. Francois, A. C. Mode of action of antibiotics on growth. World Rev. Nutr. Diet. 3, 21 (1961).

10. Visek, W. J. The mode of growth promotion by antibiotics. J. Anim. Sci. 46, 1447-1469 (1978).

11. Feighner, S. D. \& Dashkevicz, M. P. Subtherapeutic levels of antibiotics in poultry feeds and their effects on weight gain, feed efficiency, and bacterial cholyltaurine hydrolase activity. Appl. Environ. Microbiol. 53, 331-336 (1987).

12. Gaskins, H. R., Collier, C. T. \& Anderson, D. B. Antibiotics as growth promotants: mode of action. Anim. Biotechnol. 13, 29-42 (2002).

13. Knarreborg, A., Lauridsen, C., Engberg, R. M. \& Jensen, S. K. Dietary antibiotic growth promoters enhance the bioavailability of $\alpha$-tocopheryl acetate in broilers by altering lipid absorption. J. Nutr. 134, 1487-1492 (2004). 
14. Niewold, T. A. The nonantibiotic anti-inflammatory effect of antimicrobial growth promoters, the real mode of action? A hypothesis. Poult. Sci. 86, 605-609 (2007).

15. Khadem, A., Soler, L., Everaert, N. \& Niewold, T. A. Growth promotion in broilers by both oxytetracycline and Macleaya cordata extract is based on their anti-inflammatory properties. Br. J. Nutr. 112, 1110-1118 (2014).

16. Soler, L. et al. Growth promotion in pigs by oxytetracycline coincides with down regulation of serum inflammatory parameters and of hibernation-associated protein HP-27. Electrophoresis 37, 1277-1286 (2016).

17. Buret, A. G. Immuno-modulation and anti-inflammatory benefits of antibiotics: the example of tilmicosin. Can. J. Vet. Res. 74, 1-10 (2010).

18. Bosi, P. et al. Feed supplemented with 3 different antibiotics improved food intake and decreased the activation of the humoral immune response in healthy weaned pigs but had differing effects on intestinal microbiota. J. Anim. Sci. 89, 4043-4053 (2011).

19. Kaukas, A., Hinton, M. \& Linton, A. H. The effect of growth-promoting antibiotics on the faecal enterococci of healthy young chickens. J. Appl. Microbiol. 64, 57-64 (1988).

20. Engberg, R. M., Hedemann, M. S., Leser, T. D. \& Jensen, B. B. Effect of zinc bacitracin and salinomycin on intestin $/$ microflora and performance of broilers. Poult. Sci. 79, 1311-1319 (2000).

21. Dumonceaux, T. J., Hill, J. E., Hemmingsen, S. M. \& Van Kessel, A. G. Characterization of intestinal microbiot. respo se to dietary virginiamycin supplementation in the broiler chicken. Appl. Environ. Microbiol. 72, 2815-2823 (2006).

22. Pedroso, A. A. et al. Intestinal bacterial community and growth performance of chickens fed diets cont ning antibiotic oult. Sci. 85, 747-752 (2006).

23. Wise, M. G. \& Siragusa, G. R. Quantitative analysis of the intestinal bacterial community in one thre ek-o commercially $^{-}$ reared broiler chickens fed conventional or antibiotic-free vegetable-based diets. J. Appl. Micre iol. 102, 1130 49 (2007).

24. Zhou, H. et al. Appropriate chicken sample size for identifying the composition of broiler in stinal microbio a affected by dietary antibiotics, using the polymerase chain reaction-denaturing gradient gel electrophoresis techn e. Poult. S i. 86, 2541-2549 (2007).

25. Lu, J., Hofacre, C., Smith, F. \& Lee, M. D. Effects of feed additives on the development he ile teri community of the broiler chicken. Animal 2, 669-676 (2008).

26. Lin, J., Hunkapiller, A. A., Layton, A. C., Chang, Y.-J. \& Robbins, K. R. Resp nse of int al microbiota to antibiotic growth promoters in chickens. Foodborne Pathog. Dis. 10, 331-337 (2013).

27. Pourabedin, M., Xu, Z., Baurhoo, B., Chevaux, E. \& Zhao, X. Effects of m nnan rosaccharide and virginiamycin on the cecal microbial community and intestinal morphology of chickens raised under subopt. (2014).

28. Neumann, A. P. \& Suen, G. Differences in major bacterial populati in th intestines of mature broilers after feeding virginiamycin or bacitracin methylene disalicylate. J. Appl. Microbiol. 119, 1515-1

29. Huyghebaert, G., Ducatelle, R. \& Van Immerseel, F. An update on alterı es to antimicrobial growth promoters for broilers. Vet. J. 187, 182-188 (2011).

30. Lin, J. Effect of antibiotic growth promoters on intestinal $\mathrm{m}$ cr in food animals: a novel model for studying the relationship between gut microbiota and human obesity? Front. Microbiot 2, 201) .

31. Cho, I. et al. Antibiotics in early life alter the murine colonic m crobiome and adiposity. Nature 488, 621-626 (2012).

32. Cox, L. M. et al. Altering the intestinal microb: ring a criti al developmental window has lasting metabolic consequences. Cell 158, 705-721 (2014).

33. Lin, J. Antibiotic growth promoters enhan nimal duction by targeting intestinal bile salt hydrolase and its producers. Front. Microbiol. 5 (2014).

34. Cocito, C. Antibiotics of the virgir amvcin famu nibitors which contain synergistic components. Microbiol. Rev. 43, 145 (1979).

35. Van den Bogaard, A. E., Merte. L ndon N/H. \& Stobberingh, E. E. High prevalence of colonization with vancomycin-and pristinamycin-resistant ente coco. health numans and pigs in The Netherlands: is the addition of antibiotics to animal feeds to blame? J. Antimicrob. Che other. 40, 1,06 (1997).

36. George, B. A., Quarles C. Fagerber,o, D. J. Virginiamycin effects on controlling necrotic enteritis infection in chickens. Poult. Sci. 61, 447-450 (198)

37. Stutz, M. W., Job 1sons S. L. \& J M, F. R. Effects of diet and bacitracin on growth, feed efficiency, and populations of Clostridium perfringens in he intestine of broiler chicks. Poult. Sci. 62, 1619-1625 (1983).

38. Manning, J. C t al. Effect of selected antibiotics and anticoccidials on Salmonella enteritidis cecal colonization and organ invasion in Leghorn ch Avian Jis. 256-261 (1994).

39. Gong. J. et al. b. zinc bacitracin, bird age and access to range on bacterial microbiota in the ileum and caeca of broiler chick Appl. Microbiol. 104, 1372-1382 (2008).

40. Chen, ro min, G. J. Kynurenine pathway metabolites in humans: disease and healthy states. Int. J. tryptophan Res. IJTR 2, 1 (2009).

41. avis, I. ₹ Liu, A. What is the tryptophan kynurenine pathway and why is it important to neurotherapeutics? (2015).

42. Tartin, M. T., Fernandez, A. G., Fernandez, E. \& Goñalons, E. Receptors implicated in the actions of serotonin on chicken ileum IOn _. dinal smooth muscle. Life Sci. 52, 1361-1369 (1993).

cortinas, L. et al. Fatty acid content in chicken thigh and breast as affected by dietary polyunsaturation level. Poult. Sci. 83, 155-1164 (2004).

44. Calder, P. C. Omega-3 fatty acids and inflammatory processes. Nutrients 2, 355-374 (2010).

45. Federation of Animal Science Societies. Guide for the care and use of agricultural animals in research and teaching. Animal Science (2010).

46. National Research Council. Nutrient requeriments of poultry. National Academy of Sciences https://doi.org/10.1103/PhysRevB.81.041203 (1994).

47. Evans, A. M., DeHaven, C. D., Barrett, T., Mitchell, M. \& Milgram, E. Integrated, nontargeted ultrahigh performance liquid chromatography/electrospray ionization tandem mass spectrometry platform for the identification and relative quantification of the small-molecule complement of biological systems. Anal. Chem. 81, 6656-6667 (2009).

48. Reitman, Z. J. et al. Profiling the effects of isocitrate dehydrogenase 1 and 2 mutations on the cellular metabolome. Proc. Natl. Acad. Sci. 108, 3270-3275 (2011).

49. Abasht, B., Mutryn, M. F., Michalek, R. D. \& Lee, W. R. Oxidative stress and metabolic perturbations in wooden breast disorder in chickens. PLoS One 11, e0153750 (2016).

\section{Acknowledgements}

This work was supported by ARS CRIS Project 8042-32000-107-00D.

\section{Author Contributions}

U.G. and S.O., E.L. and H.L. designed the research; U.G., S.O. and H.L. conducted research; U.G. and S.O. analyzed data; U.G., S.O. and E.L.; H.L. had responsibility for content. All authors read and approved the final manuscript. All authors had no conflicts of interest. 
Additional Information

Competing Interests: The authors declare no competing interests.

Publisher's note: Springer Nature remains neutral with regard to jurisdictional claims in published maps and institutional affiliations.

(c) (i) Open Access This article is licensed under a Creative Commons Attribution 4.0 International License, which permits use, sharing, adaptation, distribution and reproduction in any medium or format, as long as you give appropriate credit to the original author(s) and the source, provide a link to the Creative Commons license, and indicate if changes were made. The images or other third party material in this article are included in the article's Creative Commons license, unless indicated otherwise in a credit line to the material. If material is not included in the article's Creative Commons license and your intended vo is not permitted by statutory regulation or exceeds the permitted use, you will need to obtain permission $d$ ectly from the copyright holder. To view a copy of this license, visit http://creativecommons.org/licenses/by/4.0/

(C) The Author(s) 2018
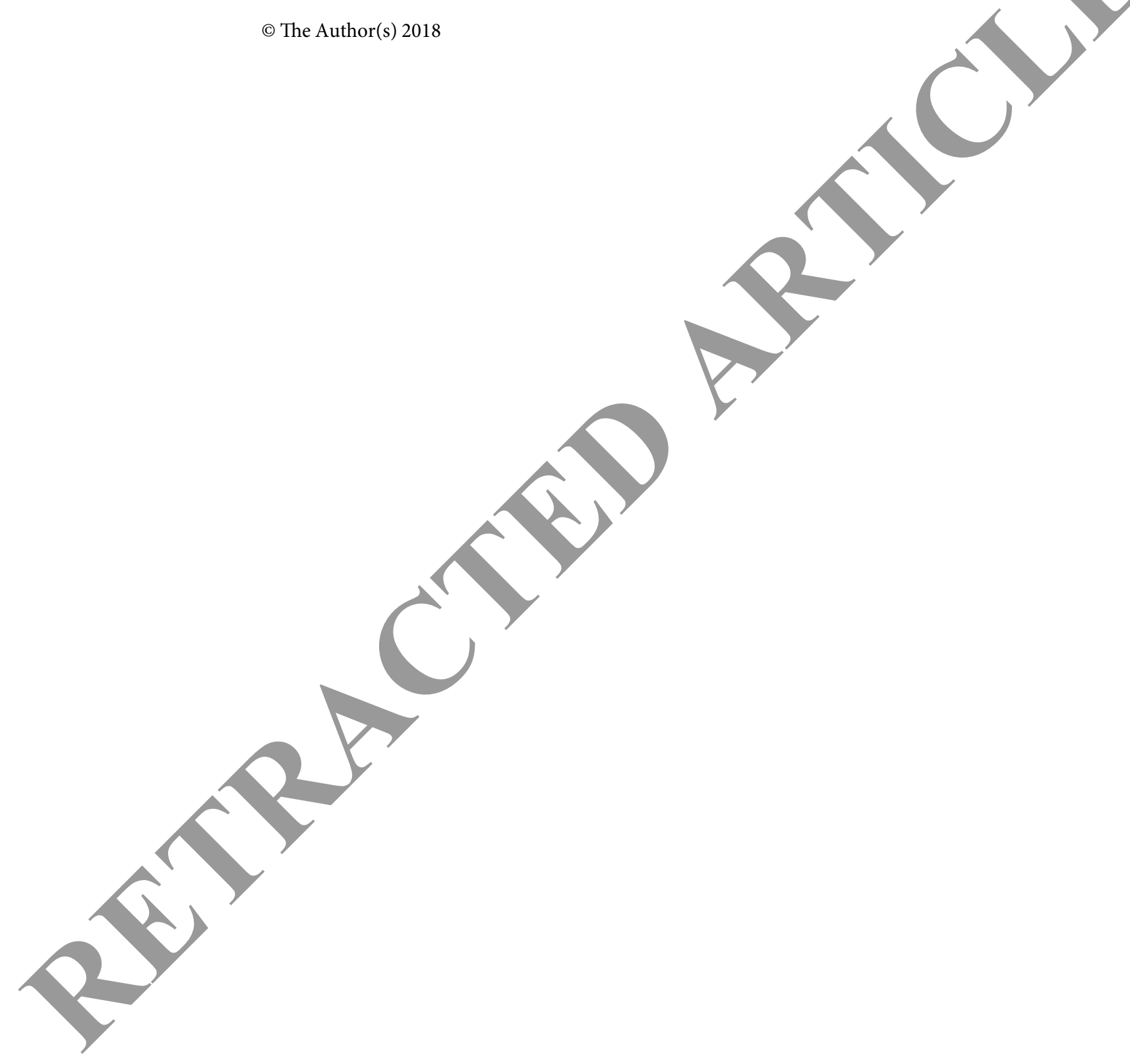